\title{
Rare kaon decay to missing energy: Implications of the NA62 result for a $Z^{\prime}$ model
}

\author{
Téssio B. de Melo, ${ }^{1, *}$ Sergey Kovalenko, ${ }^{2, \dagger}$ Farinaldo S. Queiroz, ${ }^{1,3, *}$ \\ C. Siqueira $\odot,{ }^{1,4,8}$ and Yoxara S. Villamizar $\oplus^{1,3, \|}$ \\ ${ }^{1}$ International Institute of Physics, Universidade Federal do Rio Grande do Norte, \\ Campus Universitario, Lagoa Nova, Natal-RN 59078-970, Brazil \\ ${ }^{2}$ Departamento de Ciencias Físicas, Universidad Andres Bello, Sazie 2212, Santiago, Chile \\ ${ }^{3}$ Departamento de Física, Universidade Federal do Rio Grande do Norte, Natal, RN 59078-970, Brazil \\ ${ }^{4}$ Instituto de Física de São Carlos, Universidade de São Paulo, Avenue Trabalhador São-carlense 400, \\ São Carlos 13566-590, Brazil
}

(Received 2 March 2021; accepted 5 May 2021; published 1 June 2021)

\begin{abstract}
Meson decays offer a good opportunity to probe new physics. The rare kaon decay $K^{+} \rightarrow \pi^{+} \nu \bar{\nu}$ is one of the cleanest of them and, for this reason, is rather sensitive to new physics, in particular, vector mediators. The NA62 Collaboration, running a fixed-target experiment at CERN, recently reported an unprecedented sensitivity to this decay, namely a branching fraction of $\operatorname{BR}\left(K^{+} \rightarrow \pi^{+} \nu \bar{\nu}\right)=$ $\left(11_{-3.5}^{+4.0}\right) \times 10^{-11}$ at $68 \%$ C.L. Vector mediators that couple to neutrinos may yield a sizeable contribution to this decay. Motivated by the new measurement, we interpret this result in the context of a concrete $Z^{\prime}$ model, and put our findings into perspective with the correlated $K_{L} \rightarrow \pi^{0} \nu \bar{\nu}$ decay measured by the KOTO Collaboration, current, and future colliders, namely the High-Luminosity and High-Energy Large Hadron Collider.
\end{abstract}

DOI: 10.1103/PhysRevD.103.115001

\section{INTRODUCTION}

Mesons have played a key role in the understanding of properties of elementary particles. The introduction of strangeness along with isospin lead us to the eight-fold way, based on the SU(3) flavor symmetry. These theoretical insights have contributed to the discovery of quantum chromodynamics as we know today. Another good example is the famous $\theta-\tau$ puzzle. Two different decays were found for charged strange mesons known at the time as $\theta^{+}$ and $\tau^{+}$. The decay modes had different parities, but the particles were shown to have the same mass and lifetime. It was indeed a puzzling observation. Later, it was realized that weak interactions violate parity, and these two particles were actually the same $K^{+}$meson. Additionally, the Glashow-Iliopoulos-Maiani mechanism and quark charm

\footnotetext{
*tessiomelo@gmail.com

†sergey.kovalenko@unab.cl

farinaldo.queiroz@iip.ufrn.br

§csiqueira@ifsc.usp.br

yoxara@ufrn.edu.br
}

Published by the American Physical Society under the terms of the Creative Commons Attribution 4.0 International license. Further distribution of this work must maintain attribution to the author(s) and the published article's title, journal citation, and DOI. Funded by SCOAP. surfaced as an explanation of the absence of weak flavor changing neutral currents in the processes such as $K^{+} \rightarrow \pi^{+} \nu \bar{\nu}$. The discovery of $C P$ violation in the $K^{0}-\bar{K}^{0}$ system further proved the importance of meson physics for our understanding of nature. Furthermore, meson systems are able to access possible new sources of $C P$ violation that are of paramount importance for explaining the observed baryon-antibaryon asymmetry in our universe [1]. Lastly, the $K^{+}$rare decay into neutrinos can efficiently probe the presence of heavy vector mediators, beyond the Standard Model (SM) [2-9] via the Feynman diagrams displayed in Fig. 1.

The meson decay $K^{+} \rightarrow \pi^{+} \nu \bar{\nu}$ is a flavor changing neutral current process that occurs in the SM via the box and penguin diagrams, with the latter being dominated by the top quark contribution. Due to the GIM and loop suppression, the SM contribution to this decay is very small, reading $\operatorname{BR}\left(K^{+} \rightarrow \pi^{+} \nu \bar{\nu}\right)=(8.4 \pm 1.0) \times 10^{-11}$ [10], while the NA62 currently imposes $\operatorname{BR}\left(K^{+} \rightarrow \pi^{+} \nu \bar{\nu}\right)=$ $11.0_{-3.5}^{+4.0} \times 10^{-11}[11,12]$ (results collected in 2016, 2017, and 2018). Therefore, one can clearly notice that the current sensitivity achieved by NA62 is not far from the SM prediction. Having said that, the NA62 Collaboration has been continuously searching for the rare kaon decay [12]. The KOTO Collaboration has also conducted some searches as well, but offering weaker 


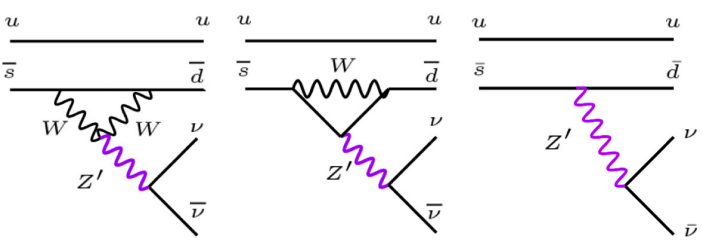

FIG. 1. Feynman diagrams that illustrate how vector mediators can contribute to the $K^{+} \rightarrow \pi^{+} \nu \bar{\nu}$ decay. The first diagram requires $Z^{\prime}$ coupling to neutrinos, the second further requires couplings to the top quark, whereas in the third case the $Z^{\prime}$ boson mediates FCNC at tree level, which is the main focus of this paper.

constraints [13]. ${ }^{1}$ To concretely show the relevance of the recent NA62 result, we will put into perspective other existing limits in a model that features a heavy vector mediator. The model is based on the $S U(3)_{C} \times S U(3)_{L} \times$ $U(1)_{Y}$ gauge group, known as 3-3-1 model. It is well motivated by the ability to naturally explain the observed replication of the generations, and nicely hosting dark matter candidates [14-21], addressing neutrino masses [22-25], among other interesting phenomena [3,26,2633 . As a result of the enlarged gauge group, the 3-3-1 model has several new gauge bosons, such as $W^{\prime}$ and $Z^{\prime}$ bosons, which are subject to restrictive constraints rising from collider physics [34-37], the muon anomalous magnetic moment [38-41], and lepton flavor violation [42,43]. We will investigate the role of the $Z^{\prime}$ gauge boson in the rate $K^{+}$decay and then use this to draw bounds on the $Z^{\prime}$ mass using the $K_{L} \rightarrow \pi^{0} \bar{\nu} \nu, K^{+} \rightarrow \pi^{+} \bar{\nu} \nu$ decays.

Our work is structured as follows: In Sec. II, we review the 3-3-1 model and compute the $Z^{\prime}$ couplings necessary to our analyses; in Sec. III, we discuss the computation of the branching fractions of the kaons in our model; in Sec. IV, we discuss our results and, finally in Sec. VI, we draw our conclusions.

\section{THE MODEL}

The 3-3-1 models are extensions of the standard model and are based on the following local symmetry group: $\mathbf{S U}(\mathbf{3})_{\mathbf{C}} \times \mathbf{S U}(\mathbf{3})_{\mathbf{L}} \times \mathbf{U}(\mathbf{1})_{\mathbf{N}}$, where $\mathbf{C}$ corresponds to the color charge, $\mathbf{L}$ denotes the left-handed fermions, and $\mathbf{N}$ is the quantum number of the $\mathrm{U}(1)$ group. The general expression for the electric charge operator in these models is written as

\footnotetext{
${ }^{1}$ KOTO collaboration has also recently reported the observation of three anomalous events in the $K_{L} \rightarrow \pi^{0} \nu \bar{\nu}$. This anomaly requires the branching ratio for this decay mode to be about 2 orders of magnitude larger than the SM one [4], indicating the presence of a new light and long-lived particle with mass of the order of $100 \mathrm{MeV}$. There is no such light particle in our model. Hence the KOTO anomaly will be regarded as a statistical fluke.
}

$$
\frac{Q}{e}=\frac{1}{2}\left(\lambda_{3}+\beta \lambda_{8}\right)+\mathrm{NI}=\left(\begin{array}{c}
\frac{1}{3}+\mathrm{N} \\
-\frac{2}{3}+\mathrm{N} \\
\frac{1}{3}+\mathrm{N}
\end{array}\right),
$$

where $\lambda_{3}=\operatorname{diag}(1,-1,0), \lambda_{8}=\operatorname{diag}(1,1,-2) / \sqrt{3}$, and I are the diagonal Gell-Mann matrices and the identity matrix, respectively. We took $\beta=-\frac{1}{\sqrt{3}}$ because in our work we choose the model known as 3-3-1 with righthanded neutrinos (3-3-1 r.h.n) [44,45]. However, we highlight that our conclusions are also applicable to the 3-3-1 model with neutral fermions proposed in [14]. The hypercharge in this model is given as

$$
Y=2 Q-\lambda_{3}=2 N-\frac{\sqrt{3} \lambda_{8}}{3},
$$

which is identical to the standard model one. The left (L)and right (R)-handed fermionic fields of this model are represented as follows:

$$
\begin{aligned}
f_{L}^{a} & =\left(\begin{array}{c}
\nu_{L}^{a} \\
\ell_{L}^{a} \\
\left(\nu_{R}^{c}\right)^{a}
\end{array}\right) \sim(1,3,-1 / 3), \\
\ell_{R}^{a} \sim(1,1,-1), & \\
Q_{i L} & =\left(\begin{array}{c}
d_{i L} \\
-u_{i L} \\
d_{i L}^{\prime}
\end{array}\right) \sim(3, \overline{3}, 0), \\
u_{i R} & \sim(3,1,2 / 3), d_{i R} \sim(3,1,-1 / 3), \\
d_{i R}^{\prime} & \sim(3,1,-1 / 3), \\
Q_{3 L} & =\left(\begin{array}{c}
u_{3 L} \\
d_{3 L} \\
T_{L}
\end{array}\right) \sim(3,3,1 / 3), \\
u_{3 R} & \sim(3,1,2 / 3), d_{3 R} \sim(3,1,-1 / 3), \\
T_{R} & \sim(3,1,2 / 3),
\end{aligned}
$$

where $a=1,2,3$ and $i=1,2$ are the generation indexes, and $f_{L}^{a}$ and $Q_{i L}, Q_{3 L}$ represent the left-handed leptonic and quark triplets, respectively. These fields encompass the SM spectrum like neutrinos $\left(\nu^{a}=\nu_{e}, \nu_{\mu}, \nu_{\tau}\right)$, charged leptons $\ell^{a}=e, \mu, \tau$, and quarks $u_{i}=\bar{u}, \bar{c}, d_{i}=\bar{d}, \bar{s}, u_{3}=t$, and $d_{3}=b$. Besides, there are other particles in addition to the SM: three right-handed neutrino $\left(\nu_{R}^{c}\right)^{a}$ and three new heavy exotic quarks $d_{i}^{\prime}$ and $T$, leading to nine leptons and nine quarks in the model. In Eqs. (3), (4), we have specified the field assignments indicating how they transform under the symmetries $\left(\mathrm{SU}(3)_{c}, \mathrm{SU}(3)_{L}, \mathrm{U}(1)_{N}\right)$, respectively. The values of their electric charge and hypercharge can be found from Eqs. (1) and (2). 
Furthermore, the 3-3-1 r.h.n model contains three scalar fields $\chi, \eta$, and $\rho$ in the following representations:

$$
\begin{aligned}
& \chi=\left(\begin{array}{c}
\chi^{0} \\
\chi^{-} \\
\chi^{\prime 0}
\end{array}\right) \sim(1,3,-1 / 3), \\
& \rho=\left(\begin{array}{c}
\rho^{+} \\
\rho^{0} \\
\rho^{\prime+}
\end{array}\right) \sim(1,3,2 / 3), \\
& \eta=\left(\begin{array}{c}
\eta^{0} \\
\eta^{-} \\
\eta^{\prime 0}
\end{array}\right) \sim(1,3,-1 / 3) .
\end{aligned}
$$

These scalar triplets in Eq. (6) are responsible for the spontaneous symmetry breaking (SSB) in the model, with the following vacuum expectation value (VEV) structure:

$$
\langle\chi\rangle=\left(\begin{array}{c}
0 \\
0 \\
v_{\chi}
\end{array}\right), \quad\langle\rho\rangle=\left(\begin{array}{c}
0 \\
v_{\rho} \\
0
\end{array}\right), \quad\langle\eta\rangle=\left(\begin{array}{c}
v_{\eta} \\
0 \\
0
\end{array}\right) .
$$

We will assume that $v_{\chi} \gg v_{\eta}, v_{\rho}$, leading to the two-step SSB,

$$
\mathbf{S U}(3)_{\mathbf{L}} \times \mathbf{U}(1)_{\mathbf{X}} \stackrel{\langle x\rangle}{\rightarrow} \mathbf{S U}(2)_{\mathbf{L}} \times \mathbf{U}(1)_{\mathbf{Y}} \stackrel{\langle\eta\rangle,\langle\rho\rangle}{\rightarrow} \mathrm{U}(1)_{\mathbf{Q}}
$$

with $U(1)_{Q}$, being the $U(1)$ from electrodynamics.

The fermion masses rise from the Yukawa Lagrangian that reads

$$
\begin{aligned}
\mathcal{L}_{Y u k}= & \lambda_{1 a} \bar{Q}_{1 L} d_{a R} \rho+\lambda_{2 i a} \bar{Q}_{i L} u_{a R} \rho^{*}+G_{a b}^{\prime} \bar{f}_{L}^{a} e_{R}^{b} \rho \\
& +G_{a b} \varepsilon^{i j k}\left(\bar{f}_{L}^{a}\right)_{i}\left(f_{L}^{b}\right)_{j}^{c}\left(\rho^{*}\right)_{k}+\lambda_{3 a} \bar{Q}_{1 L} u_{a R} \eta \\
& +\lambda_{4 i a} \bar{Q}_{i L} d_{a R} \eta^{*}+\lambda_{1} \bar{Q}_{3 L} T_{R} \chi+\lambda_{2 i j} \bar{Q}_{i L} d_{j R}^{\prime} \chi^{*} \\
& + \text { H.c. }
\end{aligned}
$$

The quark and charged lepton masses are proportional to $v=246 \mathrm{GeV}$, where $v^{2}=v_{\rho}^{2}+v_{\eta}^{2}$ similarly to the SM. The fourth term leads to a $3 \times 3$ antisymmetric neutrino mass matrix [45], which would lead to one massless and two degenerate neutrino mass eigenstates, in conflict with the neutrino oscillation data. For this reason, it is necessary to include a scalar sextet $S \sim(1,6,-2 / 3)$ in the particle content, which couples to the lepton triplet as $y_{a b} \bar{f}_{L}^{a}\left(f_{L}^{b}\right)^{c} S$. When the scalar sextet and the scalar triplet $\chi$ acquires a nonzero VEV, the 3-3-1 symmetry is broken giving rise to a scalar doublet, a singlet field, and a scalar triplet, under the SM gauge group. The active neutrino masses are proportional to $m_{\nu_{a b}} \sim\left(v_{1}+v_{2} / \Lambda\right) y_{a b}$, where $v_{1}, v_{2}$, and $\Lambda$ are the VEV of the singlet, doublet, and triplet scalars, respectively [43]. Given the freedom for the Yukawa couplings and the vacuum choices, one could easily evoke a scenario where active neutrino masses are around $0.1 \mathrm{eV}$, while right-handed neutrinos masses lie below $1 \mathrm{TeV}$, being heavy enough to not contribute to the $K \rightarrow \pi \nu \nu$ decay, but light enough to enter in the $Z^{\prime}$ width. For concreteness, doing an order of magnitude exercise, if we take $v_{1} \sim 10 \mathrm{MeV}, v_{2} \sim 100 \mathrm{GeV}$, and $\Lambda \sim 10^{10} \mathrm{GeV}$ we get $m_{\nu} \sim 0.1 \mathrm{eV}$ and $m_{\nu_{R}} \sim 100 \mathrm{GeV}$ for $y_{a b} \sim 10^{-8}$. However, one should keep in mind that indeed a right-handed neutrino may be sufficiently light to represent an additional decay channel to the $K^{+}$meson. Studies exploring this idea have been conducted by the NA62 Collaboration elsewhere [46]. Conversely, if a seesaw type I mechanism prevails, the right-handed neutrinos would not be kinematically accessible to the $Z^{\prime}$ decay, strengthening the Large Hadron Collider (LHC) bound. In this case, our reasoning is still valid but the LHC bounds will simply be a bit stronger. We highlight that this discussion concerning the weakening of the LHC bound and possible right-handed neutrino contribution to the $K^{+}$decay is also valid for the 3-31LHN model.

The gauge bosons $W$ and $Z$ acquire mass terms identical to the SM as well. In addition to the SM fields, there are new massive gauge bosons predicted by the model as a result of the enlarged gauge symmetry, denoted as $Z^{\prime}, V^{ \pm}$ and $U^{0}, U^{0 \dagger}$. The masses of these fields are

$$
\begin{aligned}
M_{W^{ \pm}}^{2} & =\frac{1}{4} g^{2} v^{2}, \quad M_{Z}^{2}=\frac{M_{W^{ \pm}}^{2}}{C_{W}^{2}}, \\
M_{Z^{\prime}}^{2} & =\frac{g^{2}}{4\left(3-4 S_{W}^{2}\right)}\left[4 C_{W}^{2} v_{\chi}^{2}+\frac{v^{2}}{C_{W}^{2}}+\frac{v^{2}\left(1-2 S_{W}^{2}\right)^{2}}{C_{W}^{2}}\right], \\
M_{V^{ \pm}}^{2} & =\frac{1}{4} g^{2}\left(v_{\chi}^{2}+v^{2}\right), M_{U^{0}}^{2}=\frac{1}{4} g^{2}\left(v_{\chi}^{2}+v^{2}\right),
\end{aligned}
$$

with $M_{W} \ll M_{U}, M_{V}, S_{W}=\sin \theta_{W}$, and $C_{W}=\cos \theta_{W}$, with $\theta_{W}$, the Weinberg angle.

The charged (CC) and neutral (NC) currents are found to be

$$
\begin{aligned}
\mathcal{L}^{C C}= & -\frac{g}{\sqrt{2}}\left[\bar{\nu}_{L}^{a} \gamma^{\mu} e_{L}^{a} W_{\mu}^{+}+\left(\nu_{R}^{\bar{c}}\right)^{a} \gamma^{\mu} e_{L}^{a} V_{\mu}^{+}+\bar{\nu}_{L}^{a} \gamma^{\mu}\left(\nu_{R}^{c}\right)^{a} U_{\mu}^{0}\right] \\
& -\frac{g}{\sqrt{2}}\left[\left(\bar{u}_{3 L} \gamma^{\mu} d_{3 L}+\bar{u}_{i L} \gamma^{\mu} d_{i L}\right) W_{\mu}^{+}\right. \\
& +\left(\bar{T}_{L} \gamma^{\mu} d_{3 L}+\bar{u}_{i L} \gamma^{\mu} d_{i L}^{\prime}\right) V_{\mu}^{+} \\
& \left.+\left(\bar{u}_{3 L} \gamma^{\mu} T_{L}-\bar{d}_{i L}^{\prime} \gamma^{\mu} d_{i L}\right) U_{\mu}^{0}+\text { H.c. }\right], \\
\mathcal{L}^{N C}= & \frac{g}{2 c_{W}}\left\{\bar{f} \gamma^{\mu}\left[a_{1 L}(f)\left(1-\gamma_{5}\right)+a_{1 R}(f)\left(1+\gamma_{5}\right)\right] f Z_{\mu}^{1}\right. \\
& \left.+\bar{f} \gamma^{\mu}\left[a_{2 L}(f)\left(1-\gamma_{5}\right)+a_{2 R}(f)\left(1+\gamma_{5}\right)\right] f Z_{\mu}^{2}\right\} .
\end{aligned}
$$

The second and third terms in Eq. (9) violate leptonic number and weak isospin [45]. $Z^{1}$ and $Z^{2}$ are neutral physical gauge bosons, which rise from the $Z$ and $Z^{\prime}$ gauge 
TABLE I. $\quad Z^{\prime}$ couplings to neutrinos and the left-handed downtype quarks, considering $\phi=0$.

\begin{tabular}{lccc}
\hline \hline & $Z^{\prime}-\nu-\bar{\nu}$ & $Z^{\prime}-\bar{d}_{i}-d_{i}$ & $Z^{\prime}-\bar{b}-b$ \\
\hline Coupling constant & $\frac{1-2 S_{W}^{2}}{2 \sqrt{3-4 S_{W}^{2}}}$ & $-\frac{\sqrt{3-4 S_{W}^{2}}}{6}$ & $\frac{3-2 S_{W}^{2}}{6 \sqrt{3-4 S_{W}^{2}}}$ \\
\hline \hline
\end{tabular}

boson mixtures. $a_{1 R}(f), a_{1 L}(f), a_{2 R}(f)$, and $a_{2 L}(f)$ are couplings of fermions with the $Z^{1}$ and $Z^{2}$ bosons. The mixing angle of these bosons is commonly denoted as $\phi$ and when $\phi=0$, the couplings of $Z^{1}$ with the leptons and quarks are the same as the boson $Z$ in the SM. Likewise, the couplings of $Z^{2}$ in this limiting case should be the same as $Z^{\prime}$ [45]. These couplings for the vertices $Z^{\prime}-\nu-\bar{\nu}$, $Z^{\prime}-\bar{d}_{i}-d_{i}$ and $Z^{\prime}-\bar{b}-b$ are shown in Table I.

In order to study meson physics in our model, and investigate its connection to the $Z^{\prime}$ boson, we need to extract the flavor changing neutral current. To do so, we start by reviewing how the flavor changing terms arise. The quark fields in the following standard rotation are

$$
\left(\begin{array}{l}
u \\
c \\
t
\end{array}\right)_{L}=V_{L}^{u}\left(\begin{array}{c}
u^{\prime} \\
c^{\prime} \\
t^{\prime}
\end{array}\right)_{L}, \quad\left(\begin{array}{l}
d \\
s \\
b
\end{array}\right)_{L}=V_{L}^{d}\left(\begin{array}{c}
d^{\prime} \\
s^{\prime} \\
b^{\prime}
\end{array}\right)_{L}
$$

where $V_{L}^{u}$ and $V_{L}^{d}$ are the $3 \times 3$ unitary matrices such that for the Cabibbo-Kobayashi-Maskawa (CKM) matrix we have $V_{\mathrm{CKM}} \equiv V_{L}^{u \dagger} V_{L}^{d}$. Note that only the left-chiral terms of the Lagrangian in Eq. (10) lead to the quark flavor violation. The right-chiral quark couplings to $Z^{\prime}$ in Eq. (10) are independent of flavor and therefore are flavor diagonal in the mass eigenstate basis. We can write these terms in the form

$$
\mathcal{L}_{Z^{\prime}} \supset \frac{g}{C_{W}}\left(\overline{D_{L}^{\prime}} \gamma^{\mu} Y_{L}^{D} D_{L}^{\prime}\right) Z_{\mu}^{\prime},
$$

with $D_{L}^{\prime}=\left(d^{\prime}, s^{\prime}, b^{\prime}\right)^{T}$, and

$$
\begin{aligned}
Y_{L}^{D}= & \frac{1}{6 \sqrt{3-4 S_{W}^{2}}} \\
& \times \operatorname{Diagonal}\left(-3+4 S_{W}^{2},-3+4 S_{W}^{2}, 3-2 S_{W}^{2}\right) .
\end{aligned}
$$

Changing the basis we get

$\mathcal{L}_{Z^{\prime}} \supset \frac{g}{C_{W}}\left(\overline{D_{L}} \gamma^{\mu} Y_{L}^{D^{\prime}} D_{L}\right) Z_{\mu}^{\prime}=\Delta_{L}^{s d}\left(\overline{s_{L}} \gamma^{\mu} d_{L}\right) Z_{\mu}^{\prime}+\cdots$,

where $D_{L}^{\prime}=V_{L}^{d} D_{L}$ and $Y_{L}^{D \prime}=V_{L}^{d \dagger} Y_{L}^{D} V_{L}^{d}$. Using the unitarity of the $V_{L}$ matrix, we finally find the coupling between the quarks $d$ and $s$, which is given by

$$
\Delta_{L}^{s d}=\frac{g C_{W}}{\sqrt{3-4 S_{W}^{2}}} V_{L 32}^{*} V_{L 31} .
$$

Analogously for the neutrino- $Z^{\prime}$ coupling we have

$$
\Delta_{L}^{\nu \bar{\nu}}=\frac{g}{2 C_{W}} \frac{1-2 S_{W}^{2}}{\sqrt{3-4 S_{W}^{2}}} .
$$

In principle, we can vary the entries of the matrix $V_{L}^{d}$ freely since the CKM matrix does not constrain $V_{L}^{d}$, but the product $V_{L}^{d} V_{L}^{u}$. So, we choose the following parametrization for the $V_{L}^{d}$ matrix [47]:

$$
V_{L}^{d}=\left(\begin{array}{ccc}
\tilde{c}_{12} \tilde{c}_{13} & \tilde{s}_{12} \tilde{c}_{23} e^{i \delta_{3}}-\tilde{c}_{12} \tilde{s}_{13} \tilde{s}_{23} e^{i\left(\delta_{1}-\delta_{2}\right)} & \tilde{c}_{12} \tilde{c}_{23} \tilde{s}_{13} e^{i \delta_{1}}+\tilde{s}_{12} \tilde{s}_{23} e^{i\left(\delta_{2}+\delta_{3}\right)} \\
-\tilde{c}_{13} \tilde{s}_{12} e^{-i \delta_{3}} & \tilde{c}_{12} \tilde{c}_{23}+\tilde{s}_{12} \tilde{s}_{13} s_{13} \tilde{s}_{23} e^{i\left(\delta_{1}-\delta_{2}-\delta_{3}\right)} & -\tilde{s}_{12} \tilde{s}_{13} \tilde{c}_{23} e^{i\left(\delta_{1}-\delta_{3}\right)}-\tilde{c}_{12} \tilde{s}_{23} e^{i \delta_{2}} \\
-\tilde{s}_{13} e^{-i \delta_{1}} & -\tilde{c}_{13} \tilde{s}_{23} e^{-i \delta_{2}} & \tilde{c}_{13} \tilde{c}_{23}
\end{array}\right),
$$

where $\tilde{s}_{i j}=\sin \tilde{\theta}_{i j}, \tilde{c}_{i j}=\cos \tilde{\theta}_{i j}$, and $\delta_{i}$ are the phases, with $i, j=1,2,3$. For our purposes, the following entries will be important:

$$
\begin{gathered}
V_{L 31}^{d}=-\tilde{s}_{13} e^{-i \delta_{1}}, \\
V_{L 32}^{d}=-\tilde{c}_{13} \tilde{s}_{23} e^{-i \delta_{2}} .
\end{gathered}
$$

Then, the product which appears in the $\Delta_{L}^{s d}$ coupling is

$$
V_{L 31}^{d} V_{L 32}^{d *}=-\tilde{s}_{13} \tilde{c}_{13} \tilde{s}_{23} e^{-i\left(\delta_{1}-\delta_{2}\right)} \equiv\left|V_{L 32}^{d *} V_{L 31}^{d}\right| e^{-i \delta}
$$

where we leave the product $\left|V_{L 32}^{d *} V_{L 31}^{d}\right|$ and the phase $\delta$ as free parameters.

\section{KAON DECAYS}

The rare Kaon decay modes $K^{+} \rightarrow \pi^{+} \nu \bar{\nu}$ and $K_{L} \rightarrow$ $\pi^{0} \nu \bar{\nu}$ are considered golden modes in flavor physics, as they are very well understood theoretically and are sensitive to new physics contributions. In the SM both decays occur only at loop level and are dominated by $Z$ penguin and box diagrams. The corresponding branching ratios have been calculated at a high precision, including NNLO QCD, electroweak corrections, and also nonperturbative and isospin breaking effects [48-52]. 
The decay $K^{+} \rightarrow \pi^{+} \nu \bar{\nu}$ is $C P$ conserving, whereas $K_{L} \rightarrow \pi^{0} \nu \bar{\nu}$ is $C P$ violating. In the 3-3-1 model, the new sources of flavor and $C P$ violation which contribute to these decays come from the $Z^{\prime}$ interactions with ordinary quarks and leptons, as discussed above. Although these couplings induce the transitions at tree level, they are suppressed by the large $Z^{\prime}$ mass.

Following the notation of Ref. [53], we can write the branching ratios for the Kaon decay modes $K \rightarrow \pi \nu \bar{\nu}$ as

$$
\begin{aligned}
& \operatorname{BR}\left(K^{+} \rightarrow \pi^{+} \nu \bar{\nu}\right) \\
& \quad=\kappa_{+}\left[\left(\frac{\operatorname{Im} X_{\text {eff }}}{\lambda^{5}}\right)^{2}+\left(\frac{\operatorname{Re} X_{\text {eff }}}{\lambda^{5}}-\bar{P}_{c}(X)\right)^{2}\right],
\end{aligned}
$$

and

$$
\operatorname{BR}\left(K_{L} \rightarrow \pi^{0} \nu \bar{\nu}\right)=\kappa_{L}\left(\frac{\operatorname{Im} X_{\text {eff }}}{\lambda^{5}}\right)^{2}
$$

In these expressions $\lambda$ denotes the Cabibbo angle, $\kappa_{+}$and $\kappa_{L}$ are given by

$$
\begin{gathered}
\kappa_{+}=(5.21 \pm 0.025) \times 10^{-11}\left(\frac{\lambda}{0.2252}\right)^{8}, \\
\kappa_{L}=(2.25 \pm 0.01) \times 10^{-10}\left(\frac{\lambda}{0.2252}\right)^{8},
\end{gathered}
$$

and $P_{c}(X)$ summarizes the charm contribution,

$$
\bar{P}_{c}(X)=\left(1-\frac{\lambda^{2}}{2}\right) P_{c}(X),
$$

with

$$
P_{c}(X)=(0.42 \pm 0.03)\left(\frac{0.2252}{\lambda}\right)^{4} .
$$

$X_{\text {eff }}$ describes the contribution of short distance physics,

$$
X_{\text {eff }}=V_{t s}^{*} V_{t d} X_{L}(K),
$$

where

$$
X_{L}(K)=\eta_{X} X_{0}\left(x_{t}\right)+\frac{\Delta_{L}^{\nu \bar{\nu}}}{g_{\mathrm{SM}}^{2} m_{Z^{\prime}}^{2}} \frac{\Delta_{L}^{s d}}{V_{t s}^{*} V_{t d}}
$$

and

$$
g_{\mathrm{SM}}^{2}=4 \frac{m_{W}^{2} G_{F}^{2}}{2 \pi^{2}}=1.78137 \times 10^{-7} \mathrm{GeV}^{-2} .
$$

The first term in Eq. (22) represents the SM contribution, which is dominated by $Z$-penguin and box diagrams, and includes QCD and electroweak corrections. The factor $\eta_{X}$ is close to unity, $\eta_{X}=0.994$, and

$$
X_{0}\left(x_{t}\right)=\frac{x_{t}}{8}\left[\frac{x_{t}+2}{x_{t}-1}+\frac{3 x_{t}-6}{\left(x_{t}-1\right)^{2}} \ln x_{t}\right],
$$

with $x_{t}=m_{t}^{2} / m_{W}^{2}$. The 3-3-1 contribution is enclosed in the second term of Eq. (22), with $\Delta_{L}^{s d}$ and $\Delta_{L}^{\nu \bar{\nu}}$ given in the Eqs. (14) and (15), respectively. If $Z^{\prime}$ is absent we have $\Delta_{L}^{\nu \bar{\nu}}=\Delta_{L}^{s d}=0$ and the SM result is recovered.

The decays $K^{+} \rightarrow \pi^{+} \nu \bar{\nu}$ and $K_{L} \rightarrow \pi^{0} \nu \bar{\nu}$ are related to each other in the SM, via isospin symmetry, since both transitions are ruled by the same short distance operator. This interdependence leads to the Grossman-Nir limit [54],

$$
\mathrm{BR}\left(K_{L} \rightarrow \pi^{0} \nu \bar{\nu}\right) \leq 4.3 \mathrm{BR}\left(K^{+} \rightarrow \pi^{+} \nu \bar{\nu}\right) .
$$

For a fixed value of $\operatorname{BR}\left(K^{+} \rightarrow \pi^{+} \nu \bar{\nu}\right)$, this theoretical bound provides an upper limit for $\operatorname{BR}\left(K_{L} \rightarrow \pi^{0} \nu \bar{\nu}\right)$, which is typically still stronger than the current experimental limits. The bound remains valid in SM extensions in which the new physics is much heavier than the Kaon mass. In particular, the 3-3-1 model obeys the bound, as we present in Fig. 2. In this plot, we made a scan over the parameters $100 \mathrm{GeV}<m_{Z^{\prime}}<10000 \mathrm{GeV}$, the module $10^{-4}<\left|V_{L 32}^{d *} V_{L 31}^{d}\right|<10^{-1}$, and the $0<\delta<\pi / 2$ phase, showing explicitly the bounds from NA62 over the $\mathrm{BR}\left(K^{+} \rightarrow \pi^{+} \nu \bar{\nu}\right)$ (blue region), the limit from KOTO (gray region), and the theoretical Grossman-Nir limit (green region).

Having presented the formulas that summarizes the predictions of the 3-3-1 model regarding meson FCNC processes, we can now discuss the implications of the experimental searches for the flavor violating Kaon decays on the parameter space of the model.

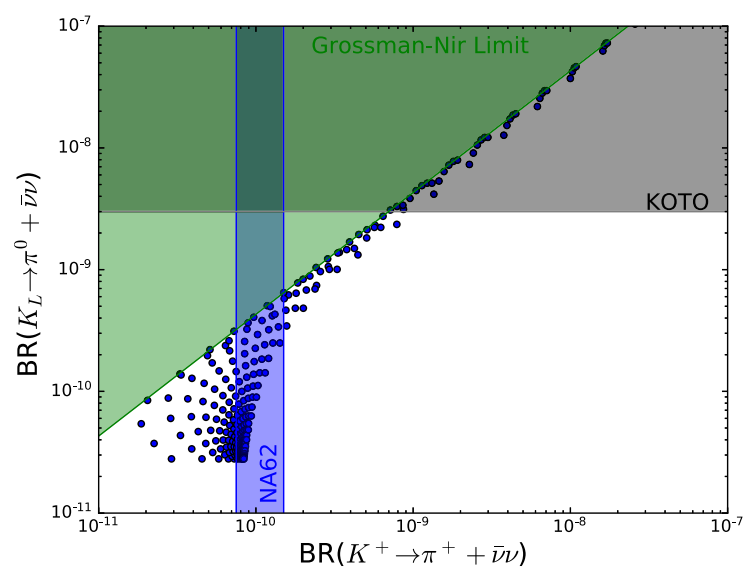

FIG. 2. $\operatorname{BR}\left(K_{L} \rightarrow \pi^{0}+\bar{\nu} \nu\right)$ versus $\operatorname{BR}\left(K^{+} \rightarrow \pi^{+}+\bar{\nu} \nu\right)$; the blue dots were generated varying the $Z^{\prime}$ mass, the module $\left|V_{L 32}^{d *} V_{L 31}^{d}\right|$, and the $\delta$ phase for the 3-3-1 r.h.n. model. We combined three different limits, the theoretical Grossman-Nir limit (green region), the NA62 bounds (blue region), and the exclusion region from KOTO (gray region). 


\section{RESULTS}

In this section, we present our results for the FCNC processes using Eqs. (20) and (21), for the 3-3-1 r.h.n model, but we emphasize that our findings are also applicable to the 3-3-1LHN [14], which is a 3-3-1 version that features heavy neutral leptons instead of right-handed neutrinos. Anyway, we compare our results with the last limits from KOTO [55] and, NA62 Run1 $(2016+2017+2018)[11,12,56]$. In Fig. 3, we display the branching for $K_{L}$ (right panel) and $K^{+}$(left panel) versus the $Z^{\prime}$ mass for the 3-3-1 r.h.n model, namely combining the standard model contribution and the new one provided by the new $Z^{\prime}$ boson, as mentioned above. We compute both branching varying the $Z^{\prime}$ mass, the phase $\delta$ (color bar) and we fix the product $\left|V_{L 32}^{d *} V_{L 31}^{d}\right|=10^{-3}$. From the left-panel of Fig. 3 one can conclude that the KOTO experiment excludes at most $Z^{\prime}$ masses around $400 \mathrm{GeV}$, which occurs for $\delta>1.4$. However, from the right panel of Fig. 3, we find that $Z^{\prime}$ masses below $3 \times$ $10^{3} \mathrm{GeV}$ might be excluded depending on the value adopted for the phase $\delta$. For $\delta \rightarrow \pi / 2$, the NA62 sensitivity to heavy $Z^{\prime}$ mediators severely weakens. Hence, NA62 yields complementary limits to other existing probes $[35,57,58]$. We can also see the presence of a dip due to destructive interference between the $Z^{\prime}$ and the SM contributions, where the branching ratio lies bellow the SM predicted value. In the plot, it occurs for $Z^{\prime}$ masses in the 600-900 GeV range, but in general its depth and location depends on the combination of $\left|V_{L 32}^{d *} V_{L 31}^{d}\right|$ and $\delta$. Notice that there is no dip when $\delta=\pi / 2$, while it reaches its maximum depth for $\delta=0$.

We have drawn quantitative conclusions based on a particular value for $V_{L 32}^{d *} V_{L 31}^{d}$. To assess how our bounds change for different choices of this product we examine the range $10^{-4}<V_{L 32}^{d *} V_{L 31}^{d}<10^{-1}$. In Fig. 4 we show exclusion plots in the plane $\left|V_{L 32}^{d *} V_{L 31}^{d}\right|$ versus $m_{Z^{\prime}}$, for fixed $\delta=0$ (left panel) and $\delta=\pi / 2$ (right panel). Here we combined limits from NA62, KOTO, and LHC, High-Luminosity LHC (HL-LHC), and High-Energy LHC (HE-LHC). The strongest collider bounds on the $Z^{\prime}$ mass stems from the resonant production of $Z^{\prime}$ decaying into dileptons [40].

There is an interesting interplay between collider and flavor physics. While collider bounds rely mostly on the interaction strength between the $Z^{\prime}$ boson and fermions, which is fixed in 3-3-1 models, the flavor bounds weaken with $\left|V_{L 32}^{d *} V_{L 31}^{d}\right|$. The collider bounds displayed in Fig. 4 are conservative, as they take into account the presence of $Z^{\prime}$ decays into right-handed neutrinos and exotic quarks, which can be light enough so that the decays are not kinematically forbidden. The original lower mass bound reads $m_{Z^{\prime}}>4 \mathrm{TeV}$ [57], but those exotic decays were ignored in [57]. If more decay channels become available, then the bound weakens. Here, in order to take into account this uncertainty on the $Z^{\prime}$ decay modes, we assume conservatively a branching fraction of $50 \%$ into charged leptons, which leads to the gray region in Fig. 4 in agreement with [41]. We also show the prospects for the HL-LHC, with $3 \mathrm{ab}^{-1}$ of integrated luminosity, and the HELHC, which corresponds to an integrated luminosity of $15 \mathrm{ab}^{-1}$ at a center-of-mass energy of $27 \mathrm{TeV}$. These projected collider limits were obtained using the code described in [59], which can be used to forecast lower mass limits for resonance searches, which is precisely our case.

We found that the NA62 bounds from the decay $K^{+} \rightarrow$ $\pi^{+} \nu \bar{\nu}$ are rather restrictive, providing stronger limits than the ones from colliders over a significant region of the parameter space. Looking at the left-panel of Fig. 4, for instance, where $\delta=0$, NA62 can exclude $Z^{\prime}$ masses as high
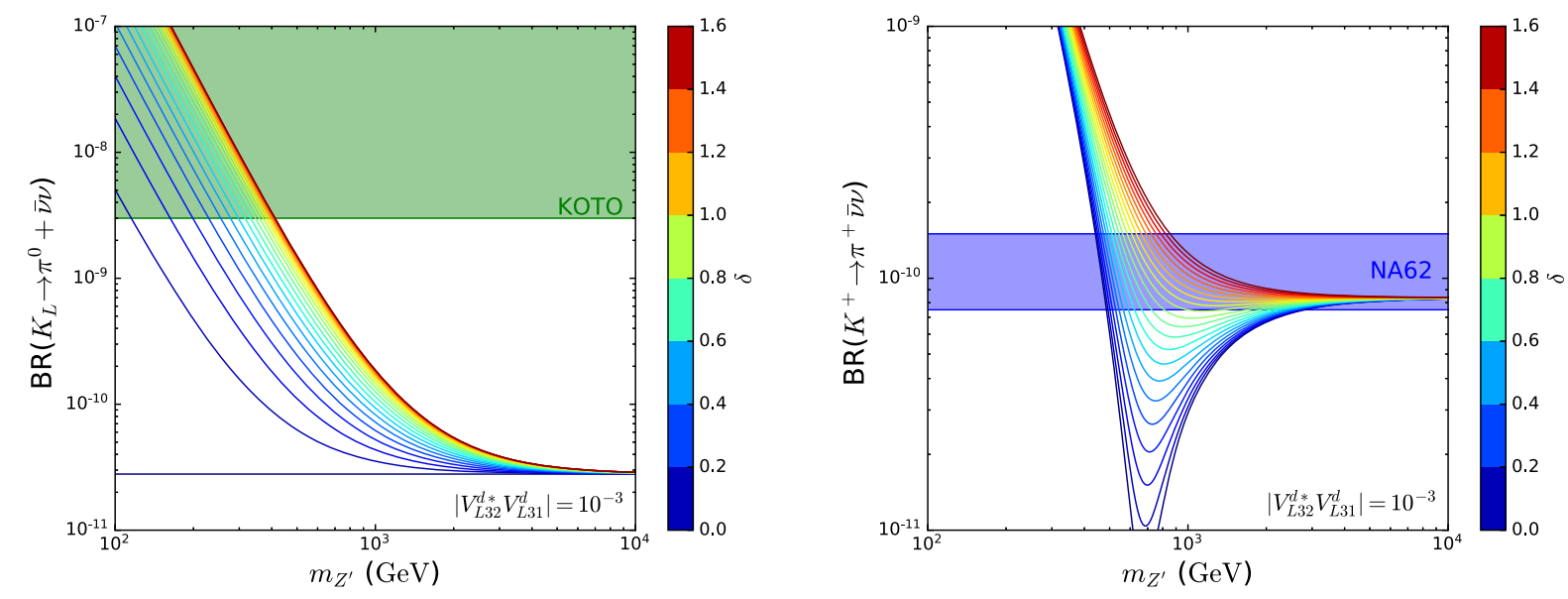

FIG. 3. Branching ratio versus $Z^{\prime}$ mass for kaon long (left panel), $\mathrm{BR}\left(K_{L} \rightarrow \pi^{0}+\bar{\nu} \nu\right.$ ), and for the $K^{+}, \mathrm{BR}\left(K^{+} \rightarrow \pi^{+}+\bar{\nu} \nu\right)$ (right panel). The green band provides the excluded region by the KOTO experiment, and the blue region provides that allowed by the NA62 experiment. The colored bar represents the variation of the $\delta$ phase; for details see the text. 

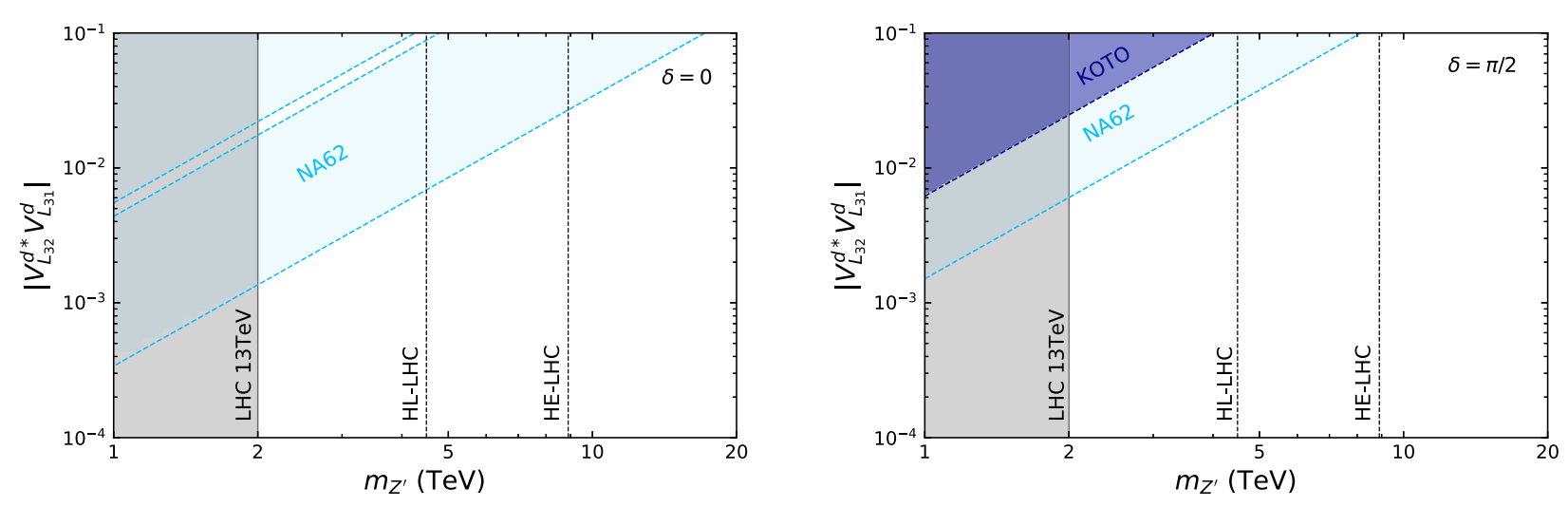

FIG. 4. Excluded parameter space regions in the 3-3-1 r.h.n model, in the plane $\left|V_{L 32}^{d *} V_{L 31}^{d}\right|$ versus $m_{Z^{\prime}}$, for $\delta=0$ (left) and $\delta=\pi / 2$ (right). The colored regions represent the limits from NA62 (cyan), KOTO (blue) and LHC current (gray), and prospects (dashed lines).

as $10 \mathrm{TeV}$, if $\left|V_{L 32}^{d *} V_{L 31}^{d}\right|$ is of the order $10^{-2}$ or larger. In the same vein, NA62 enforces the product $\left|V_{L 32}^{d *} V_{L 31}^{d}\right|$ to remain below $\sim 10^{-3}$, when $m_{Z^{\prime}} \sim 2 \mathrm{TeV}$.

On the other hand, these parameters are completely unconstrained by KOTO when $\delta=0$, since the contribution from $Z^{\prime}$ to the decay $K_{L} \rightarrow \pi^{0} \nu \bar{\nu}$ vanishes in this case. In the absence of new $C P$ violating sources, the $\operatorname{BR}\left(K_{L} \rightarrow\right.$ $\left.\pi^{0} \nu \bar{\nu}\right)$ takes the same value as in the SM, since this process will occur only through the contribution to $C P$ violation from the SM via CKM matrix.

This can be easily understood with Eq. (21), from which we see that $\operatorname{BR}\left(K_{L} \rightarrow \pi^{0} \nu \bar{\nu}\right)$ depends only on the imaginary part of $X_{\text {eff }}$, in particular, on $\operatorname{Im}\left(V_{t s}^{*} V_{t d}\right)$ when $\delta=0$. Still in the left panel of Fig. 4 we notice that in the range shown, the KOTO limit does not appear; as $\delta$ increases from 0 to $\pi / 2$, this bound becomes more relevant, and the KOTO exclusion region gradually grows, reflecting the enhancement in the $K_{L} \rightarrow \pi^{0} \nu \bar{\nu}$ decay, while the NA62 exclusion region slightly decreases. Nevertheless, even with maximum enhancement at $\delta=\pi / 2$ (right plot), the KOTO bounds remain less constraining compared to NA62.

\section{DISCUSSION}

Our conclusions relied on the presence of flavor changing interactions involving the $Z^{\prime}$ boson, but as the model features a large scalar sector, that could be new sources of flavor changing interactions rising from the heavy scalar fields. These new contributions have been shown to be subdominant, and thus can be safely ignored. Also, regarding the possible impact of the presence of righthanded neutrinos on the $K \rightarrow \pi \nu \nu$ decays, they can give some contribution if their masses are of order $\mathrm{MeV}$. However, this is not the case in this model, where they get masses above the $\mathrm{GeV}$ scale, so their presence does not alter our results. Moreover, although the entire reasoning was based on the 3-3-1 model with right-handed neutrinos, our results are also applicable to the 3-3-1 model where the right-handed neutrinos are replaced by heavy neutral fields. This occurs because these models have the same neutral current. In summary, our findings are relevant for two different models and solid irrespective of the presence of scalar fields.

\section{CONCLUSION}

In this work we explored the FCNC processes mediated by a $Z^{\prime}$ gauge boson featuring both in the 3-3-1 r.h.n and 33-1LHN models. We computed the $K^{+}$and $K_{L}$ decay rates to missing energy, considering the extra contributions from the $Z^{\prime}$ in addition to the SM contribution, leaving the quark mixing matrix and the $Z^{\prime}$ mass as free parameters. We performed a complementary analysis using the results from NA62, KOTO, and the LHC (current and prospects) to set bounds on the 3-3-1 r.h.n parameters. We found that the last result of the NA62 experiment was able to constrain a large region of the parameter space, setting lower limits on the $Z^{\prime}$ mass that can be more stringent than those from dilepton searches at the LHC. For example, we can impose $m_{Z^{\prime}}>$ $10 \mathrm{TeV}$ for $\left|V_{L 32}^{*} V_{L 31}\right| \sim 10^{-1}$, while $\left|V_{L 32}^{*} V_{L 31}\right| \lesssim 2 \times$ $10^{-3}$ for $m_{Z^{\prime}}=3 \mathrm{TeV}$. These results apply for $\delta=0$, where the sensitivity of NA62 is maximum. In the case when the new $C P$ violation effects are large our constraints weaken.

\section{ACKNOWLEDGMENTS}

The authors thank Antonio Santos and Diego Cogollo for discussions. T. M., C. S., and F. S. Q. thank UFRN and MEC for financial support. F. S. Q. also acknowledges the CNPq Grants No. 303817/2018-6 and No. 421952/2018-0, the financial support from ICTP-SAIFR FAPESP Grant No. 2016/01343-7, and the Serrapilheira Institute (Grant No. Serra-1912-31613). F. S. Q. and C. S. have been supported by the São Paulo Research Foundation (FAPESP) through Grant No. 2015/15897-1. C. S. is 
supported by Grant No. 2020/00320-9, São Paulo Research Foundation (FAPESP). S. K. acknowledges the support of the FONDECYT-Chile Grant No. 1190845, ANID-Chile PIA/APOYO AFB180002.
Y. S. V. acknowledges the financial support from CAPES under Grant No. 88882.375870/2019-01. We thank the High Performance Computing Center (NPAD) at UFRN for providing computational resources.
[1] M. Dine and A. Kusenko, The origin of the matterantimatter asymmetry, Rev. Mod. Phys. 76, 1 (2003).

[2] D. Cogollo, A. de Andrade, F. Queiroz, and P. Rebello Teles, Novel sources of flavor changed neutral currents in the $331_{R H N}$ model, Eur. Phys. J. C 72, 2029 (2012).

[3] F. S. Queiroz, C. Siqueira, and J. W. F. Valle, Constraining flavor changing interactions from LHC Run-2 dilepton bounds with vector mediators, Phys. Lett. B 763, 269 (2016).

[4] T. Kitahara, T. Okui, G. Perez, Y. Soreq, and K. Tobioka, New Physics Implications of Recent Search for $K_{L} \rightarrow \pi^{0} \nu \bar{\nu}$ at KOTO, Phys. Rev. Lett. 124, 071801 (2020).

[5] D. Borah, L. Mukherjee, and S. Nandi, Low scale U(1) gauge symmetry as an origin of dark matter, neutrino mass and flavour anomalies, J. High Energy Phys. 12 (2020) 052.

[6] B. Dutta, S. Ghosh, and T. Li, Explaining $(g-2)_{\mu, e}$, the KOTO anomaly and the MiniBooNE excess in an extended Higgs model with sterile neutrinos, Phys. Rev. D 102, 055017 (2020).

[7] Y. Jho, S. M. Lee, S. C. Park, Y. Park, and P.-Y. Tseng, Light gauge boson interpretation for $(g-2)_{\mu}$ and the $\mathrm{K}_{L} \rightarrow$ $\pi^{0}+$ (invisible) anomaly at the J-PARC KOTO experiment, J. High Energy Phys. 04 (2020) 086.

[8] J. Aebischer, A. J. Buras, and J. Kumar, Another SMEFT story: $Z^{\prime}$ facing new results on $\varepsilon^{\prime} / \varepsilon, \Delta M_{K}$ and $K \rightarrow \pi \nu \bar{\nu}$, J. High Energy Phys. 12 (2020) 097.

[9] Z. Kang and Y. Shigekami, $(g-2)_{\mu}$ versus $K \rightarrow \pi+E_{\text {miss }}$ induced by the $(B-L)_{23}$ boson, J. High Energy Phys. 04 (2021) 238.

[10] A. J. Buras, D. Buttazzo, J. Girrbach-Noe, and R. Knegjens, $K^{+} \rightarrow \pi^{+} \nu \bar{\nu}$ and $K_{L} \rightarrow \pi^{0} \nu \bar{\nu}$ in the standard model: Status and perspectives, J. High Energy Phys. 11 (2015) 033.

[11] R. Marchevski, New result on the search for the $k^{+} \rightarrow \pi^{+} \bar{\nu} \nu$ decay at the na62 experiment at cern, in 40th International Conference on High Energy physics (2020), https://pos.sissa .it/390/398/pdf.

[12] E. Cortina Gil et al. (NA62 Collaboration), An investigation of the very rare $K^{+} \rightarrow \pi^{+} \nu \bar{\nu}$ decay, J. High Energy Phys. 11 (2020) 042.

[13] J. Ahn et al. (KOTO Collaboration), Study of the $K_{L} \rightarrow$ $\pi^{0} \nu \bar{\nu}$ Decay at the J-PARC KOTO Experiment, Phys. Rev. Lett. 126, 121801 (2021).

[14] J. Mizukoshi, C. de S.Pires, F. Queiroz, and P. Rodrigues da Silva, WIMPs in a 3-3-1 model with heavy sterile neutrinos, Phys. Rev. D 83, 065024 (2011).

[15] C. Kelso, C. A. de S. Pires, S. Profumo, F. S. Queiroz, and P.S. Rodrigues da Silva, A 331 WIMPy dark radiation model, Eur. Phys. J. C 74, 2797 (2014).
[16] S. Profumo and F. S. Queiroz, Constraining the $Z^{\prime}$ mass in 331 models using direct dark matter detection, Eur. Phys. J. C 74, 2960 (2014).

[17] D. Cogollo, A. X. Gonzalez-Morales, F. S. Queiroz, and P. R. Teles, Excluding the light dark matter window of a 331 model using LHC and direct dark matter detection data, J. Cosmol. Astropart. Phys. 11 (2014) 002.

[18] J. Montero, A. Romero, and B. Sánchez-Vega, Axion dark matter in a 3-3-1 model, Phys. Rev. D 97, 063015 (2018).

[19] C. Carvajal, B. Sánchez-Vega, and O. Zapata, Linking axionlike dark matter to neutrino masses, Phys. Rev. D 96, 115035 (2017).

[20] E. Abdalla et al., Brazilian community report on dark matter, arXiv:1912.10076.

[21] J. Leite, A. Morales, J. W. Valle, and C. A. Vaquera-Araujo, Dark matter stability from Dirac neutrinos in scotogenic 3-3-1-1 theory, Phys. Rev. D 102, 015022 (2020).

[22] V. Vien, H. Long, and A. Cárcamo Hernández, Lepton masses and mixings in a $T^{\prime}$ flavoured 3-3-1 model with type I and II seesaw mechanisms, Mod. Phys. Lett. A 34, 1950005 (2019).

[23] T. Nguyen, T. T. Le, T. Hong, and L. Hue, Decay of standard model-like Higgs boson $h \rightarrow \mu \tau$ in a 3-3-1 model with inverse seesaw neutrino masses, Phys. Rev. D 97, 073003 (2018).

[24] A. Cárcamo Hernández, N. A. Pérez-Julve, and Y. Hidalgo Velásquez, Fermion masses and mixings and some phenomenological aspects of a 3-3-1 model with linear seesaw mechanism, Phys. Rev. D 100, 095025 (2019).

[25] A. Cárcamo Hernández, Y. Hidalgo Velásquez, and N. A. Pérez-Julve, A 3-3-1 model with low scale seesaw mechanisms, Eur. Phys. J. C 79, 828 (2019).

[26] P. Van Dong, N. Ngan, T. Tham, L. Thien, and N. Thuy, Phenomenology of the simple 3-3-1 model with inert scalars, Phys. Rev. D 99, 095031 (2019).

[27] A. Alves, G. Arcadi, P. Dong, L. Duarte, F. S. Queiroz, and J. W. F. Valle, Matter-parity as a residual gauge symmetry: Probing a theory of cosmological dark matter, Phys. Lett. B 772, 825 (2017).

[28] P. Dong, D. Huong, F. S. Queiroz, J. W. F. Valle, and C. Vaquera-Araujo, The dark side of flipped trinification, J. High Energy Phys. 04 (2018) 143.

[29] D. Huong, D. Dinh, L. Thien, and P. Van Dong, Dark matter and flavor changing in the flipped 3-3-1 model, J. High Energy Phys. 08 (2019) 051.

[30] G. Arcadi, M. Lindner, J. Martins, and F. S. Queiroz, New physics probes: Atomic parity violation, polarized electron scattering and neutrino-nucleus coherent scattering, Nucl. Phys. B959, 115158 (2020). 
[31] D. Van Loi, C. H. Nam, and P. Van Dong, Dark matter in the fully flipped 3-3-1-1 model, arXiv:2012.10979.

[32] N. Duy, T. Inami, and D. Huong, Physical constraints derived from FCNC in the 3-3-1-1 model, arXiv:2009.09698.

[33] A. G. Dias, J. Leite, B. Sánchez-Vega, and W. C. Vieira, Dynamical symmetry breaking and fermion mass hierarchy in the scale-invariant 3-3-1 model, Phys. Rev. D 102, 015021 (2020).

[34] Q.-H. Cao and D.-M. Zhang, Collider phenomenology of the 3-3-1 model, arXiv:1611.09337.

[35] A. Nepomuceno and B. Meirose, Limits on 331 vector bosons from LHC proton collision data, Phys. Rev. D 101, 035017 (2020).

[36] A. Nepomuceno, B. Meirose, G. Marvila, and M. Viera, Exclusion limits on neutral, singly and doubly charged vector bosons at LHC, Proc. Sci. EPS-HEP2019 (2020) 553.

[37] Y.-B. Liu, A.-Q. An, and H.-M. Han, The 3-3-1 model with $\mathrm{RH}$ neutrinos and associated $\mathrm{Z} \mathrm{H}$ production at high-energy e+ e- collider, Braz. J. Phys. 41, 66 (2011).

[38] C. Kelso, P. Pinheiro, F. S. Queiroz, and W. Shepherd, The muon anomalous magnetic moment in the reduced minimal 3-3-1 model, Eur. Phys. J. C 74, 2808 (2014).

[39] C. Kelso, H. Long, R. Martinez, and F. S. Queiroz, Connection of $g-2_{\mu}$, electroweak, dark matter, and collider constraints on 331 models, Phys. Rev. D 90, 113011 (2014).

[40] A. S. de Jesus, S. Kovalenko, C. A. de S. Pires, F. S. Queiroz, and Y.S. Villamizar, Dead or alive? Implications of the muon anomalous magnetic moment for 3-3-1 models, Phys. Lett. B 809, 135689 (2020).

[41] A. De Jesus, S. Kovalenko, F. Queiroz, C. Siqueira, and K. Sinha, Vectorlike leptons and inert scalar triplet: Lepton flavor violation, $g-2$, and collider searches, Phys. Rev. D 102, 035004 (2020).

[42] G. Arcadi, C. Ferreira, F. Goertz, M. Guzzo, F. S. Queiroz, and A. Santos, Lepton flavor violation induced by dark matter, Phys. Rev. D 97, 075022 (2018).

[43] M. M. Ferreira, T. B. de Melo, S. Kovalenko, P. R. Pinheiro, and F.S. Queiroz, Lepton flavor violation and collider searches in a type I + II seesaw model, Eur. Phys. J. C 79, 955 (2019).

[44] H. N. Long, SU(3)-L x U(1)-N model for right-handed neutrino neutral currents, Phys. Rev. D 54, 4691 (1996).

[45] H. N. Long, The 331 model with right handed neutrinos, Phys. Rev. D 53, 437 (1996).
[46] C. Lazzeroni et al. (NA62 Collaboration), Search for heavy neutrinos in $K^{+} \rightarrow \mu^{+} \nu_{\mu}$ decays, Phys. Lett. B 772, 712 (2017).

[47] A. J. Buras, F. De Fazio, J. Girrbach, and M. V. Carlucci, The anatomy of quark flavour observables in 331 models in the flavour precision era, J. High Energy Phys. 02 (2013) 023.

[48] G. Buchalla and A. J. Buras, Two loop large $m_{t}$ electroweak corrections to $K \rightarrow \pi \nu \bar{\nu}$ for arbitrary Higgs boson mass, Phys. Rev. D 57, 216 (1998).

[49] J. Brod, M. Gorbahn, and E. Stamou, Two-loop electroweak corrections for the $K \rightarrow \pi \nu \bar{\nu}$ decays, Phys. Rev. D 83, 034030 (2011).

[50] A. Buras, M. Gorbahn, U. Haisch, and U. Nierste, Rare Decay $K^{+} \rightarrow \pi^{+} \nu \bar{\nu}$ at the Next-to-Next-to-Leading Order in QCD, Phys. Rev. Lett. 95, 261805 (2005).

[51] A. J. Buras, M. Gorbahn, U. Haisch, and U. Nierste, Charm quark contribution to $K^{+} \rightarrow \pi^{+} \nu \bar{\nu}$ at next-to-next-to-leading order, J. High Energy Phys. 11 (2006) 002; Erratum, J. High Energy Phys. 11 (2012) 167.

[52] J. Brod and M. Gorbahn, Electroweak corrections to the charm quark contribution to $K^{+} \rightarrow \pi^{+} \nu \bar{\nu}$, Phys. Rev. D 78, 034006 (2008).

[53] A. J. Buras and J. Girrbach, Towards the identification of new physics through quark flavour violating processes, Rep. Prog. Phys. 77, 086201 (2014).

[54] Y. Grossman and Y. Nir, $K_{L} \rightarrow \pi^{0} \nu \bar{\nu}$ beyond the standard model, Phys. Lett. B 398, 163 (1997).

[55] J. Ahn et al. (KOTO Collaboration), Search for the $K_{L} \rightarrow$ $\pi^{0} \nu \bar{\nu}$ and $K_{L} \rightarrow \pi^{0} X^{0}$ Decays at the J-PARC KOTO Experiment, Phys. Rev. Lett. 122, 021802 (2019).

[56] R. Marchevski, Evidence for the decay $K^{+} \rightarrow \pi+\bar{\nu} \nu$ from the NA62 experiment at CERN, https://indico.cern.ch/event/ 868940/contributions/3815641/attachments/2080353/ 3496097/RadoslavMarchevski_ICHEP_2020.pdf (2020).

[57] M. Lindner, M. Platscher, and F. S. Queiroz, A call for new physics: The muon anomalous magnetic moment and lepton flavor violation, Phys. Rep. 731, 1 (2018).

[58] A. C. O. Santos and P. Vasconcelos, Lower mass bound on the $W^{\prime}$ mass via neutrinoless double beta decay in a 3-3-1 model, Adv. High Energy Phys. 2018, 9132381 (2018).

[59] A. Thamm, R. Torre, and A. Wulzer, Future tests of Higgs compositeness: Direct vs indirect, J. High Energy Phys. 07 (2015) 100. 